\title{
Matrix metalloproteinase-3 in brain physiology and neurodegeneration
}

\author{
Anna Maria Lech ${ }^{1,2, D-F}$, Grzegorz Wiera ${ }^{2, D-F}$, Jerzy Władysław Mozrzymas ${ }^{2, D-F}$ \\ 1 Department of Molecular Physiology and Neurobiology, University of Wrocław, Poland \\ ${ }^{2}$ Laboratory of Neuroscience, Department of Biophysics, Wroclaw Medical University, Poland \\ A - research concept and design; $\mathrm{B}$ - collection and/or assembly of data; $\mathrm{C}$ - data analysis and interpretation; \\ $D$ - writing the article; $E$ - critical revision of the article; $F$ - final approval of the article
}

\section{Address for correspondence \\ Anna Maria Lech \\ E-mail:anna.lech@uwr.edu.pl \\ Funding sources \\ National Science Centre, Poland, \\ grant No. 2017/26/D/NZ4/00450 \\ Conflict of interest \\ None declared}

Received on January 28, 2019

Reviewed on February 28, 2019

Accepted on June 27, 2019

Published online on December 16, 2019

\begin{abstract}
Structural and functional synapse reorganization is one of the key issues of learning and memory mechanisms. Specific proteases, called matrix metalloproteinases (MMPs), play a pivotal role during learning-related modification of neural circuits. Different types of MMPs modify the extracellular perisynaptic environment, leading to the plastic changes in the synapses. In recentyears, there has been an increasing interest in the role played by matrix metalloproteinase-3 (MMP-3) in various processes occurring in the mammalian brain, both in physiological and pathological conditions. In this review, we discuss a crucial function of MMP-3 in synaptic plasticity, learning, neuronal development, as well as in neuroregeneration. We discuss the involvement of MMP-3 in synaptic long-term potentiation, which is likely to have a profound impact on experiencedependent learning. On the other hand, we also provide examples of deleterious actions of uncontrolled MMP-3 activity on the central nervous system (CNS) and its contribution to Alzheimer's and Parkinson's diseases (AD and PD). Since the molecular mechanisms controlled by MMP-3 have a profound and diverse impact on physiological and pathological brain functioning, their deep understanding may be crucial for the development of more specific methods for the treatment of neuropsychiatric diseases.
\end{abstract}

Key words: neuroplasticity, MMP-3, neurodegenerative diseases, learning and memory, long-term potentiation

Cite as

Lech AM, Wiera G, Mozrzymas JW. Matrix metalloproteinase-3 in brain physiology and neurodegeneration. Adv Clin Exp Med. 2019;28 1717-1722. doi:10.17219/acem/110319

DOI

10.17219/acem/110319

\section{Copyright}

Copyright by Author(s)

This is an article distributed under the terms of the

Creative Commons Attribution 3.0 Unported (CC BY 3.0)

(https://creativecommons.org/licenses/by/3.0/) 


\section{Introduction}

Learning and memory are contingent on a continuous modification of neural connectivity and circuitry by means of mechanisms that depend on network activity. This ability to change the synaptic strength in an experience-dependent manner is called synaptic plasticity and is considered as a substrate of learning, memory and behavior. ${ }^{1}$ As a result of synaptic plasticity, the strength of synaptic transmission can be either enhanced or depressed. The plasticity that leads to the stable amplification of synaptic transmission is called long-term potentiation (LTP). Conversely, in the process of long-term depression (LTD) synaptic strength is reduced. Upon learning, both inhibitory and excitatory synapses undergo synaptic plasticity. ${ }^{1}$ Furthermore, the balance between excitation and inhibition is a key feature in the proper functioning of the brain and the disruption in this dynamic "equilibrium" may underlie different neurological diseases such as autism ${ }^{2,3}$; schizophrenia ${ }^{3}$ or epilepsy. ${ }^{4}$

Both structural and functional reorganization of the synapse starts to occur at early stages of central nervous system (CNS) development and continue to take place in the adult learning brain in the form of complex processes of neuroplasticity. It is well established that synapses are surrounded by a complex network of extracellular matrix (ECM) constituents, ${ }^{5,6}$ including structural proteins, proteoglycans, glycosaminoglycans, adhesion molecules as well as growth factors. A growing body of experimental evidence suggests that diverse molecules in the ECM play crucial physiological roles in synaptogenesis, ${ }^{7,8}$ synaptic plasticity ${ }^{9,10}$ and regeneration of CNS after injury. ${ }^{11}$ Moreover, the structure of brain ECM is not static as it is continuously remodeled, e.g., by proteolysis of ECM proteins that leads to the modifications in the synapse proteome and its interaction with the extracellular environment. Degradation of ECM proteins and peptides is catalyzed, in particular, by serine proteases (such as tPA or neuropsin) and matrix metalloproteinases (MMPs). In recent years, numerous studies described the crucial role played by MMPs-dependent ECM proteolysis in physiological processes such as synaptic plasticity, learning and memory. ${ }^{12,13}$ Nonetheless, abnormal expression, localization and activity of MMPs could have a detrimental impact on brain functioning, ${ }^{6,14,15}$ and, in physiological conditions, MMPs activity is kept under strict control at both the transcriptional and cellular levels by controlling the synthesis, release, activation, inhibition, and degradation.

The main purpose of this review is to discuss recent research into the matrix metalloproteinase-3 (MMP-3), one of the crucial brain proteases engaged both in physiological brain functions and in pathogenesis of neurological diseases. In the first section, we present a brief description of the MMP family. Then, we discuss the beneficial involvement of MMP-3 in key physiological and regenerative processes in $\mathrm{CNS}$. We highlight the specific MMP-3-dependent proteolysis of ECM components and its role in development, synaptic plasticity, learning and memory, as well as in neuroregeneration after CNS injury. In the final chapter, we focus on the role of MMP-3 in pathophysiological conditions.

\section{Matrix metalloproteinases and MMP-3}

Matrix metalloproteinase-3 and, in general, MMPs have been identified as the major brain proteases affecting numerous processes. Because of the broad substrate spectrum, MMP-3 initially was considered primarily in relation to its role in pathophysiology. More recently, however, several physiological functions of this MMP have been revealed. Indeed, as we outline in the present review, MMP-3 plays a crucial role in the mammalian brain development, synaptic plasticity, learning, and memory.

Matrix metalloproteinases constitute a part of the larger family of proteases called metzincin clan. These multidomain, proteolytic enzymes, with a zinc ion $\left(\mathrm{Zn}^{2+}\right)$ in their catalytic site, can degrade ECM components, growth factors and adhesion molecules. Therefore, they are characterized by the ability to modify the extracellular environment and to modulate cell-to-cell signaling. In humans, MMP family consists of 24 distinct members, which are expressed either as soluble or transmembrane proteins. ${ }^{16}$ Based on ECM substrate specificity and domain composition, MMPs are organized into 6 groups: collagenases, gelatinases, stromelysins, matrilysins, transmembrane MMPs, and "others". ${ }^{16}$ Numerous studies have shown that the proteolytic activity of MMPs is extracellular, but their intracellular action was also suggested (MMP-3, ${ }^{17}$ MMP-12 18 .

A number of studies have found that MMPs in CNS play a fundamental role in synaptic plasticity ${ }^{19,20}$ and dendritic spine morphology. ${ }^{21}$ Furthermore, MMPs are able to regulate cell proliferation and differentiation, ${ }^{22}$ migration, ${ }^{23}$ inflammation, ${ }^{24}$ and apoptosis. ${ }^{25,26}$ Thus, not surprisingly, MMPs are precisely controlled. In the CNS, MMPs are expressed and secreted both by neurons and glial cells ${ }^{13}$ but their activity in normal conditions is low. The level of MMP activity significantly increases only in specific time windows, for example, during the induction of synaptic plasticity or repairing processes. Because MMPs are secreted mainly in their inactive form called zymogen, proMMPs in the extracellular space are inactive until the interaction between catalytic zinc ion and the cysteine residue at pro-peptide domain is disturbed. Matrix metalloproteinases can be activated by other proteases or, e.g., by reactive oxygen species (ROS). To block MMP activity, proteases are degraded or inhibited. Tissue inhibitors of metalloproteinases (TIMPs) bind noncovalently to MMP (in 1:1 ratio), which blocks its activity. 
It has been shown that a precisely balanced level of TIMP activity is essential, as its alterations may lead to various pathologies, including different types of cancer. ${ }^{27}$

Matrix metalloproteinase-3 belongs to stromelysin group of MMPs. Numerous studies have shown that MMP-3 can function both extra- and intracellularly. Extracellularly, proMMP-3 may be activated by the serine proteinases like plasmin or other MMPs. ${ }^{28}$ Matrix metalloproteinase-3 cleaves ECM components such as aggrecan, tenascins, fibronectin, laminin ${ }^{29}$; tumor necrosis factor $\alpha$ (TNF- $\alpha$ ) precursor; interleukin $1 b$ (IL-1b) and pro-forms of other MMPs like proMMP-1, proMMP-3 and proMMP-9. ${ }^{29}$ As already mentioned, MMP-3 proteolytic activity was also reported inside the cells. Choi et al. described proMMP-3 activation inside the dopaminergic neurons that undergo cellular stress, in which pro-MMP-3 zymogen was activated by serine proteases. ${ }^{25}$ Furthermore, there are several studies showing that exogenous MMP-3 can enter the cytoplasm, probably through clathrin-dependent endocytosis, ${ }^{30}$ and may even translocate into the nucleus. ${ }^{17,31}$ The proper balance of MMP-3 activity is regulated by TIMP-1 and altered MMP-3 activity connected with lower level of TIMP-1 plays an important role, e.g., in neuronal apoptosis during oxidative stress. ${ }^{32}$

\section{Matrix metalloproteinase-3 in the developing CNS}

Among others, Vaillant et al. described increased expression of MMP-3 and MMP-9 in dividing granular precursor cells in the developing cerebellum. ${ }^{33}$ During this period MMP-3 and other MMPs are crucial for cell migration and neurite outgrowth through the degradation or activation of cell surface proteins and ECM components. ${ }^{33}$ Moreover, MMP-3 has also been shown to play a crucial role in dendritogenesis. Reduction in dendritic tree size of Purkinje cells in the cerebellum was described both in postnatal, juvenile and adult $m m p$-3-deficient mice $\left(\mathrm{MMP}^{-} 3^{-/-}\right) .{ }^{34}$ These findings can be explained by the interaction of MMP-3 with repellent or attractive signaling molecules in the extracellular environment. Likewise, studies conducted on MMP-3-deficient mice shed light on the effects of MMP-3 on the morphology of pyramidal neurons in cortex and hippocampus. The Golgi-stained MMP- $3^{-/-}$pyramidal cells from layer $\mathrm{V}$ of the visual cortex exhibit significantly shorter apical dendrites compared to the wild-type controls. ${ }^{35}$ On the contrary, Nowak et al. have shown that there are no significant differences between morphology of $\mathrm{MMP}-3^{-/-}$pyramidal neurons in the CA1 hippocampal field in comparison to the wild-type group. ${ }^{36}$ Collectively, these studies suggest that the functioning of the MMP-3 in developing CNS is more complex and may depend on the region of the brain.

\section{Matrix metalloproteinase-3 in synaptic plasticity, learning and memory}

The architecture of the synapse is determined by both cell-cell and cell-ECM interactions. The coordinated, activity-dependent remodeling of the perisynaptic extracellular environment regulates synaptic transmission and synaptic plasticity. ${ }^{14}$ Over the past decade or so, the growing body of evidence has indicated that MMP-9 is a key player in neuroplasticity mechanisms. Although the functioning of MMP-9 in neural circuits has been extensively described, ${ }^{19,37}$ more recent works also highlighted the impact of MMP-3 on CNS plasticity. In the hippocampal Schaffer collaterals-CA1 pathway, one can distinguish 2 different LTP components: 1) dependent on NMDA receptors (nmdaLTP); 2) dependent on L-type voltagedependent $\mathrm{Ca}^{2+}$ channels (VDCCs, vdccLTP). In 2002, Evers et al. have described an impairment of vdccLTP in the hippocampus of tenascin- $\mathrm{C}$ deficient mice. ${ }^{10} \mathrm{Be}$ cause tenascin-C is a well-known MMP-3 substrate, ${ }^{38}$ it may suggest the involvement of MMP-3 in vdccLTP. Additionally, Kochlamazashvili et al. demonstrated that vdccLTP is dependent on the presence of hyaluronic acid in ECM in the vicinity of synapses, because enzymatic digestion of hyaluronan impaired specifically vdccLTP. ${ }^{39}$ These results reveal a new "dimension" of the synaptic plasticity, namely that enzymatic modification (such as proteolysis) of ECM constituents plays a pivotal role in regulating plastic phenomena at a variety of synapses. Moreover, recent studies have confirmed that MMP-3 activity is indeed crucial in regulating vdcc-dependent form of LTP in hippocampus, but not for nmdaLTP (that is regulated, e.g., by MMP-9).$^{13}$ Nevertheless, there is also some evidence that MMP-3 can affect NMDA receptors. In cultured spinal cord neurons, chronic NMDAR overstimulation leads to an increase in MMP-3 activity which, in turn, cleaves GluN1 subunit of NMDA receptor to constrain calcium influx and prevent neuron apoptosis. ${ }^{40}$ The activity of MMP-3 is also crucial for the plasticity of neuronal excitability. Brzdak et al. ${ }^{41}$ have suggested that potentiation of neuronal spiking requires short-term activation of MMP-3, which, in turn, increases calcium influx into neurons through NMDA receptors. Additionally, Brzdak et al. clearly demonstrated that MMP-3 activity differs between basal and apical dendrites of pyramidal neurons from the CA1 region of the hippocampus and the plasticity of synapses located in apical dendrites are MMP-3-dependent in contrast to those in basal dendrites. ${ }^{42}$ Finally, contrary to LTP, the role of MMP-3 on LTD is still unknown.

The impact of MMP-3 on learning and memory has not been studied in detail. There is limited evidence that during hippocampus-dependent learning (e.g., in Morris water maze), the level of MMP-3 activity and mRNA are increased 
within the first days after experiment. ${ }^{43}$ This could indicate that a higher level of MMP-3 as well as MMP-9 is important over a relatively short period, in which plasticity phenomena are induced. So far, however, the exact role of MMP-3 in learning remains unknown. To understand the broader aspect of MMP-3 functioning in behavior, experiments using $\mathrm{MMP}-3^{-/-}$mice are necessary. Although the physiological role of MMP-3 still awaits a thorough and extensive investigation, the available evidence points to its key role in neural plasticity and development.

\section{Matrix metalloproteinase-3 in CNS after injury}

Several studies have reported the involvement of MMP-3 in neurite outgrowth and remyelinisation. Gonthier et al. have noted that MMP-3 can be engaged in the elongation of axons in response to specific attractor molecules. ${ }^{44}$ More recent studies have reported that MMP-3 as well as others MMPs can overcome chondroitin sulphate proteoglycans (CSPGs)-dependent inhibition of neurite regeneration. ${ }^{45}$ Chondroitin sulphate proteoglycans are produced and secreted during glial scar formation to effectively slow down or even block the axonal regeneration. Importantly, almost all CSPGs constituents are putative substrates of MMP-3. Cua et al. have observed that local expression of MMP-3 in the vicinity of glial scar is increased and MMP-3 activity promotes axonal outgrowth. ${ }^{45}$ Furthermore, a few years earlier Pizzi and Crowe have reported that the application of fibroblasts overexpressing MMP-3 into the injured spinal cord augments axon regrowth. ${ }^{46}$ They have additionally observed transient progression in motor functions recovery, what could have been related to axonal regeneration. Another aspect of beneficial functions of MMP-3 is related to remyelination. Cuprizone-induced demyelination is the most often examined model in which remyelination process can be assessed. Immunohistochemical investigation showed a significant increase in the expression level of MMP-3 during remyelination in the corpus callosum induced by cuprizone. ${ }^{47}$ Altogether, these studies provide important insights into the protective roles played by the MMP-3 activity after injury and are likely to indicate promising avenues for future therapeutic developments.

\section{The role of MMP-3 in neuronal pathologies}

Besides crucial role played by MMP-3 in brain physiology, its excessive uncontrolled activity often correlates with numerous neurodegenerative disorders. In several pathologies, MMP-3 appears to have a causative role, which may be related to the disruption of the blood-brain barrier (BBB), role in demyelination, cell apoptosis or initiation of the inflammatory response. In subsequent sections, we describe the role played by MMP-3 in the pathophysiology of Alzheimer's and Parkinson's diseases (AD and PD).

\section{MMP-3 in Parkinson's disease}

Numerous reports indicate the involvement of MMP-3 in the pathophysiology of PD, which is characterized by a progressive loss of dopaminergic neurons in the substantia nigra of the midbrain, leading to cognitive and motor disorders. The increase in the level of MMP-3 protein and activity has been observed in various experimental models of PD such as in animals injected with 6-hydroxydopamine or lipopolysaccharide. ${ }^{48}$ In the case of MPTP (1-methyl-4-phenyl-1,2,3,6-tetrahydropyridine)-induced PD, a significant reduction in the degeneration of the $s u b$ stantia nigra neurons has been observed in mice deficient in MMP-3 compared to wild-type controls. ${ }^{49}$ Similarly, studies on tissue cultures also have shown that cell death of dopaminergic neurons may be limited by inhibition of MMP-3 or by MMP-3 knock-out. ${ }^{50}$ Substantia nigra is particularly rich in microglial cells; therefore, the role of MMP-3 in the pathophysiology of PD may be related to the activation of microglia and inflammatory processes in response to cellular stress. ${ }^{51}$ Additionally, changes in the permeability of the blood-brain barrier were observed both in PD patients and in animal PD models. ${ }^{52}$ Tight junction proteins are among MMP-3 putative substrates, thus the MMP-3-dependent increase in BBB permeability is considered as one of PD pathomechanisms.

Additionally, it is also worth noting that cellular proteins whose mutations correlates with PD are also MMP-3 substrates. The dysfunction of $\alpha$-synuclein, which is a component of Lewy bodies, is a well-known player in PD pathogenesis. Matrix metalloproteinase-3 cleaves the $C$-terminal part of $\alpha$-synuclein, exposing a hydrophobic patch on the protein surface, which contributes to the increased formation of protein aggregates. ${ }^{51}$ It has also been shown that mutations in the $\alpha$-synuclein, which are associated with early onset of PD, lead to an increased occurrence of $\alpha$-synuclein in Lewy bodies. This, in turn, suggests that the mutant protein may be more prone to proteolysis mediated by MMP-3. Another aspect of MMP-3 engagement in PD pathogenesis is related to protein DJ-1 that protects from oxidative proteasomal and mitochondrial stress. Mutations in DJ-1 protein and its loss of activity have been observed in PD patients. Therefore, because DJ-1 is a substrate for MMP-3, the decreased activity of DJ-1 in PD may be a result of increased uncontrolled MMP-3 proteolytic activity. ${ }^{53}$ In conclusion, the role of MMP-3 in the pathophysiology of PD is manifested through the contribution of protease to the formation of $\alpha$-synuclein aggregates and by the interference of the MMP-3 protease with the protective functions of DJ-1 protein against oxidative stress. 


\section{MMP-3 in Alzheimer's disease}

Matrix metalloproteinase-3 contributes also to the development of $A D$, which is one of the most common neurodegenerative disorders and the main cause of dementia. The main characteristic features of AD are a widespread degeneration of neurons, the formation of so-called plaques that contain amyloid- $\beta(\mathrm{A} \beta)$ peptide and deposition of neurofibrillary tangles abnormally rich in hyperphosphorylated tau protein. ${ }^{14}$ Amyloid- $\beta$ is the product of proteolytic cleavage of amyloid precursor protein (APP). The involvement of MMP-3 in AD pathogenesis is complex. Firstly, it was shown that patients suffering from AD have an elevated level of MMP-3 in the brain, especially in the white matter and senile plaques. ${ }^{54}$ It is suggested that the expression of MMP-3 in microglial cells and astrocytes can be induced by the $\mathrm{A} \beta$ peptide. ${ }^{55}$ Secondly, the analysis of $m m p-3$ gene polymorphisms has linked the $6 \mathrm{~A}$ allele of the $m m p-3$ gene promoter $(-16125 \mathrm{~A} / 6 \mathrm{~A})$ to the higher susceptibility to AD. ${ }^{56}$ This result was; however, contested by others. ${ }^{57}$ Finally, it was reported that in the human cerebrospinal fluid the reduction of MMP-3 level goes hand in hand with a lower level of $A \beta .{ }^{58}$ Additionally, in patients with risk markers for $\mathrm{AD}$, a higher MMP-3 levels and a higher MMP-3/TIMP-1 ratio were reported in comparison to those without risk markers. ${ }^{59}$ Moreover, it was proposed that MMP-3 plasma levels could be a biomarker for early diagnosis of AD. ${ }^{60}$

\section{Conclusions}

The examples discussed above underscore many roles played by MMP-3 in the developing and adult CNS. Although, over the years, more attention has been directed to the function of MMP-3 in pathophysiological processes, recent research suggests that, in the hippocampus and cortex, MMP-3 regulates also synaptic plasticity and takes part in the processes of CNS recovery after injury. In particular, the activation of MMP-3 is essential for the induction of long-term potentiation dependent on voltagegated calcium channels. ${ }^{10,13,41}$ Additionally, plastic changes at synapses correlate with short-term window of MMP-3 activity in hippocampus. ${ }^{43}$ Consistently, the activity of MMP-3 is also important in the developing brain, ${ }^{33}$ and has been shown to play a pivotal role in the dendritogenesis of pyramidal neurons in the cortex and Purkinje cells in the cerebellum. ${ }^{34}$

On the other hand, uncontrolled MMP-3 activity is often associated with neurodegenerative disorders. As was mentioned before, numerous authors described the involvement of MMP-3 in PD and AD. Matrix metalloproteinase- 3 has been found to be engaged in the pathological process of dopaminergic neurons loss in the substantia nigra. ${ }^{49}$ In addition, MMP-3 contributes to the increased formation of $\alpha$-synuclein aggregates in PD patients. ${ }^{52}$
In the same way, recent research reported an elevated level of MMP-3 in brains of AD patients, ${ }^{55,56,60}$ and proposed the use of MMP-3 as a promising biomarker for AD diagnosis. ${ }^{60}$ Taking into consideration the involvement of excessive MMP-3 activity in CNS pathophysiology, the MMP-3 inhibitors may have potential clinical applications. Overall, it should be noted that increasing our knowledge about precise brain molecular mechanisms dependent on MMP-3 and its specific substrates can consequently lead to the development of new methods for the treatment of PD and AD.

\section{ORCID iDs}

Anna Maria Lech (D) https://orcid.org/0000-0001-8550-4803 Grzegorz Wiera (D) https://orcid.org/0000-0003-2489-4451 Jerzy Władysław Mozrzymas (D) https://orcid.org/0000-0003-1674-1818

\section{References}

1. Citri A, Malenka RC. Synaptic plasticity: Multiple forms, functions, and mechanisms. Neuropsychopharmacology. 2008;33(1):18-41.

2. Uzunova G, Pallanti S, Hollander E. Excitatory/inhibitory imbalance in autism spectrum disorders: Implications for interventions and therapeutics. World J Biol Psychiatry. 2016;17(3):174-186.

3. Canitano R, Pallagrosi M. Autism spectrum disorders and schizophrenia spectrum disorders: Excitation/inhibition imbalance and developmental trajectories. Front Psychiatry. 2017;8:1-7.

4. Buckley AH, Holmes GL. Epilepsy and autism. Cold Spring Harb Perspect Med. 2016;6(4):a022749.

5. Dityatev A, Schachner M. Extracellular matrix molecules and synaptic plasticity. Nat Rev Neurosci. 2003;4(6):456-468.

6. Huntley GW. Synaptic circuit remodelling by matrix metalloproteinases in health and disease. Nat Rev Neurosci. 2012;13(11):743-757.

7. Allen NJ, Bennett ML, Foo LC, et al. Astrocyte glypicans 4 and 6 promote formation of excitatory synapses via GluA1 AMPA receptors. Nature. 2012;486(7403):410-414.

8. Dityatev A. Polysialylated neural cell adhesion molecule promotes remodeling and formation of hippocampal synapses. J Neurosci. 2004;24(42):9372-9382.

9. Albiñana E, Gutierrez-Luengo J, Hernández-Juarez N, et al. Chondroitin sulfate induces depression of synaptic transmission and modulation of neuronal plasticity in rat hippocampal slices. Neural Plast. 2015;2015:463854.

10. Evers MR, Salmen B, Bukalo O, et al. Impairment of L-type Ca2 channel-dependent forms of hippocampal synaptic plasticity in mice deficient in the extracellular matrix glycoprotein tenascin-C. J Neurosci. 2002;22(16):7117-7194.

11. Wang D, Ichiyama RM, Zhao R, Andrews MR, Fawcett JW. Chondroitinase combined with rehabilitation promotes recovery of forelimb function in rats with chronic spinal cord injury. J Neurosci. 2011;31(25): 9332-9344.

12. Al-Muhtasib N, Forcelli PA, Conant KE, Vicini S. MMP-1 overexpression selectively alters inhibition in D1 spiny projection neurons in the mouse nucleus accumbens core. Sci Rep. 2018;8(1):16230.

13. Wiera G, Nowak D, van Hove I, Dziegiel P, Moons L, Mozrzymas JW. Mechanisms of NMDA receptor- and voltage-gated L-type calcium channel-dependent hippocampal LTP critically rely on proteolysis that is mediated by distinct metalloproteinases. J Neurosci. 2017;37(5):1240-1256.

14. Brzdak P, Nowak D, Wiera G, Mozrzymas JW. Multifaceted roles of metzincins in CNS physiology and pathology: From synaptic plasticity and cognition to neurodegenerative disorders. Front Cell Neurosci. 2017;11:1-22.

15. Dubey D, McRae PA, Rankin-Gee EK, et al. Increased metalloproteinase activity in the hippocampus following status epilepticus. Epilepsy Res. 2017;132:50-58.

16. Nagase H, Visse R, Murphy G. Structure and function of matrix metalloproteinases and TIMPs. Cardiovasc Res. 2006;69(3):562-573. 
17. Si-Tayeb K, Monvoisin A, Mazzocco C, et al. Matrix metalloproteinase 3 is present in the cell nucleus and is involved in apoptosis. Am J Pathol. 2006;169(4):1390-1401.

18. Dandachi NG, Shapiro SD. A protean protease: MMP-12 fights viruses as a protease and a transcription factor. Nat Med. 2014;20(5):470-472.

19. Nagy V. Matrix metalloproteinase- 9 is required for hippocampal latephase long-term potentiation and memory. J Neurosci. 2006;26(7): 1923-1934.

20. Brzdąk P, Włodarczyk J, Mozrzymas JW, Wójtowicz T. Matrix metalloprotease 3 activity supports hippocampal EPSP-to-Spike plasticity following patterned neuronal activity via the regulation of NMDAR function and calcium flux. Mol Neurobiol. 2017;54(1):804-816.

21. Magnowska M, Gorkiewicz T, Suska A, et al. Transient ECM protease activity promotes synaptic plasticity. Sci Rep. 2016;6:27757.

22. Valente MM, Allen M, Bortolotto V, Lim ST, Conant K, Grilli M. The MMP-1/PAR-1 axis enhances proliferation and neuronal differentiation of adult hippocampal neural progenitor cells. Neural Plast. 2015;2015:646595.

23. Endo K, Takino T, Miyamori H, et al. Cleavage of syndecan-1 by membrane type matrix metalloproteinase-1 stimulates cell migration. J Biol Chem. 2003;278(42):40764-40770.

24. Marchant DJ, Bellac CL, Moraes TJ, et al. A new transcriptional role for matrix metalloproteinase-12 in antiviral immunity. Nat Med. 2014; 20(5):493-502.

25. Choi DH, Kim E-M, Son HJ, et al. A novel intracellular role of matrix metalloproteinase-3 during apoptosis of dopaminergic cells. J Neurochem. 2008;106(1):405-415.

26. Biswas MHU, Almeida S, Lopez-Gonzalez R, et al. MMP-9 and MMP-2 contribute to neuronal cell death in iPSC models of frontotemporal dementia with MAPT mutations. Stem Cell Rep. 2016;7(3):316-324.

27. Jackson HW, Defamie V, Waterhouse P, Khokha R. TIMPs: Versatile extracellular regulators in cancer. Nat Rev Cancer. 2017;17(1):38-53.

28. Nagase H, Enghild JJ, Suzuki K, Salvesen G. Stepwise activation mechanisms of the precursor of matrix metalloproteinase 3 (stromelysin) by proteinases and (4-aminophenyl)mercuric acetate. Biochemistry. 1990;29(24):5783-5789.

29. Nagase H, Fields CG, Fields GB. Design and characterization of a fluorogenic substrate selectively hydrolyzed by stromelysin 1 (matrix metalloproteinase-3). J Biol Chem. 1994;269(33):20952-20957.

30. Traub LM. Tickets to ride: Selecting cargo for clathrin-regulated internalization. Nat Rev Mol Cell Biol. 2009;10(9):583-596.

31. Eguchi T, Kubota S, Kawata K, et al. Novel transcription factor-like function of human matrix metalloproteinase 3 regulating the CTGF/ CCN2 gene. Mol Cell Biol. 2008;28(7):2391-2413.

32. Choi D-H, Kim J-H, Seo J-H, Lee J, Choi WS, Kim Y-S. Matrix metalloproteinase-3 causes dopaminergic neuronal death through nox 1 regenerated oxidative stress. PLoS One. 2014;9(12):e115954.

33. Vaillant C, Didier-Bazès M, Hutter A, Belin M-F, Thomasset N. Spatiotemporal expression patterns of metalloproteinases and their inhibitors in the postnatal developing rat cerebellum. J Neurosci. 1999; 19(12):4994-5004.

34. Van Hove I, Verslegers M, Buyens T, et al. An aberrant cerebellar development in mice lacking matrix metalloproteinase-3. Mol Neurobiol. 2012;45(1):17-29.

35. Aerts J, Nys J, Moons L, Hu T-T, Arckens L. Altered neuronal architecture and plasticity in the visual cortex of adult MMP-3-deficient mice. Brain Struct Funct. 2015;220(5):2675-2689.

36. Nowak D, De Groef $L$, Moons L, Mozrzymas JW. MMP-3 deficiency does not influence the length and number of CA1 dendrites of hippocampus of adult mice. Acta Neurobiol Exp (Wars). 2018;78(3):281-286.

37. Wiera G, Wozniak G, Bajor M, Kaczmarek L, Mozrzymas JW. Maintenance of long-term potentiation in hippocampal mossy fiberCA3 pathway requires fine-tuned MMP-9 proteolytic activity. Hippocampus. 2013;23(6):529-543.

38. Imai K, Kusakabe M, Sakakura T, Nakanishi I, Okada Y. Susceptibility of tenascin to degradation by matrix metalloproteinases and serine proteinases. FEBS Lett. 1994;352(2):216-218

39. Kochlamazashvili G, Henneberger C, Bukalo O, et al. The extracellular matrix molecule hyaluronic acid regulates hippocampal synaptic plasticity by modulating postsynaptic L-type Ca2+channels. Neuron. 2010;67(1):116-128.
40. Pauly T, Ratliff M, Pietrowski E, et al. Activity-dependent shedding of the NMDA receptor glycine binding site by matrix metalloproteinase 3: A putative mechanism of postsynaptic plasticity. PLoS One. 2008;3:e2681.

41. Brzdak P, Włodarczyk J, Mozrzymas JW, Wójtowicz T. Matrix metalloprotease 3 activity supports hippocampal EPSP-to-spike plasticity following patterned neuronal activity via the regulation of NMDAR function and calcium flux. Mol Neurobiol. 2017;54(1):804-816.

42. Brzdak P, Wójcicka O, Zareba-Koziol M, et al. Synaptic potentiation at basal and apical dendrites of hippocampal pyramidal neurons involves activation of a distinct set of extracellular and intracellular molecular cues. Cereb Cortex. 2019;29(1):283-304.

43. Meighan SE, Meighan PC, Choudhury P, et al. Effects of extracellular matrix-degrading proteases matrix metalloproteinases 3 and 9 on spatial learning and synaptic plasticity. J Neurochem. 2006;96: 1227-1241.

44. Gonthier B, Nasarre C, Roth L, et al. Functional interaction between matrix metalloproteinase-3 and semaphorin-3C during cortical axonal growth and guidance. Cereb Cortex. 2007;17:1712-1721.

45. Cua RC, Lau LW, Keough MB, Midha R, Apte SS, Yong VW. Overcoming neurite-inhibitory chondroitin sulfate proteoglycans in the astrocyte matrix. Glia. 2013;61:972-984.

46. Pizzi MA, Crowe MJ. Transplantation of fibroblasts that overexpress matrix metalloproteinase-3 into the site of spinal cord injury in rats. J Neurotrauma. 2006;23:1750-1765.

47. Hoyos HC, Marder M, Ulrich R, et al. The role of galectin-3: From oligodendroglial differentiation and myelination to demyelination and remyelination processes in a cuprizone-induced demyelination model. Adv Exp Med Biol. 2016;949:311-332.

48. McClain JA, Phillips LL, Fillmore HL. Increased MMP-3 and CTGF expression during lipopolysaccharide-induced dopaminergic neurodegeneration. Neurosci Lett. 2009;460(1):27-31.

49. Kim YS, Choi DH, Block ML, et al. A pivotal role of matrix metalloproteinase-3 activity in dopaminergic neuronal degeneration via microglial activation. FASEB J. 2007;21(1):179-187.

50. Choi DH, Kim EM, Son HJ, et al. A novel intracellular role of matrix metalloproteinase-3 during apoptosis of dopaminergic cells. J Neurochem. 2008;106(1):405-415.

51. Kim EM, Hwang O. Role of matrix metalloproteinase-3 in neurodegeneration. J Neurochem. 2011;116(1):22-32.

52. Kortekaas R, Leenders KL, van Oostrom JC, et al. Blood-brain barrier dysfunction in parkinsonian midbrain in vivo. Ann Neurol. 2005;57(2): 176-179.

53. Choi DH, Kim YJ, Kim YG, Joh TH, Beal MF, Kim YS. Role of matrix metalloproteinase 3-mediated alpha-synuclein cleavage in dopaminergic cell death. J Biol Chem. 2011;286(16):14168-14177.

54. Yoshiyama $Y$, Asahina $M$, Hattori T. Selective distribution of matrix metalloproteinase-3 (MMP-3) in Alzheimer's disease brain. Acta Neuropathol. 2000;99(2):91-95.

55. Deb S, Gottschall PE. Increased production of matrix metalloproteinases in enriched astrocyte and mixed hippocampal cultures treated with beta-amyloid peptides. J Neurochem. 1996;66(4):1641-1647.

56. Baig S, Kehoe PG, Love S. MMP-2,-3 and -9 levels and activity are not related to Abeta load in the frontal cortex in Alzheimer's disease. Neuropathol Appl Neurobiol. 2008;34(2):205-215.

57. Reitz C, van Rooij FJ, de Maat MP, et al. Matrix metalloproteinase 3 haplotypes and dementia and Alzheimer's disease. The Rotterdam Study. Neurobiol Aging. 2008;29(6):874-881.

58. Mlekusch R, Humpel C. Matrix metalloproteinases- 2 and -3 are reduced in cerebrospinal fluid with low beta-amyloid1-42 levels. Neurosci Lett. 2009;466(3):135-138.

59. Stomrud E, Björkqvist M, Janciauskiene S, Minthon L, Hansson O. Alterations of matrix metalloproteinases in the healthy elderly with increased risk of prodromal Alzheimer's disease. Alzheimers Res Ther. 2010;2(3):20-20.

60. Peng M, Jia J, Qin W. Plasma gelsolin and matrix metalloproteinase 3 as potential biomarkers for Alzheimer disease. Neurosci Lett. 2015; 595:116-121. 